\title{
Botulinum Toxin Complex: A Delivery Vehicle of Botulinum Neurotoxin Traveling Digestive Tract
}

\author{
Yoshimasa Sagane, Ken Inui, Shin-Ichiro Miyashita, Keita Miyata, \\ Tomonori Suzuki, Koichi Niwa and Toshihiro Watanabe
}

Additional information is available at the end of the chapter

http://dx.doi.org/10.5772/46023

\section{Introduction}

Botulinum neurotoxin (BoNT) produced by an anaerobic bacterium Clostridium botulinum causes highly fatal disease called botulism. BoNT is a zinc-dependent metalloprotease [1] with a molecular mass of $150 \mathrm{kDa}$, and classified into seven distinct serotypes A through G. Serotype A, B, E and F BoNTs dominantly cause human botulism, whereas serotype C and D BoNTs are the causative substance of the animal and avian botulism [2,3]. The BoNT invades into the human or animal body via one of three ways; toxin production by the bacterium colonized on the digestive tract in early childhoods (infant botulism), entrance of the toxin from the wound (wound botulism), and the oral ingestion of the toxin-contaminated foods (food-borne botulism). The food-borne botulism is the most frequent among the three cases. In any of these cases, the BoNT ultimately reaches the neuromuscular junction and enters nerve cell via receptor-mediated endocytosis. Once in the nerve cell, the metalloprotease activity of the toxin cleaves the specific site on the target proteins associated with the intracellular vesicle transport, such as synapse-associated protein of molecular weight 25,000 (SNAP-25; serotypes A, C and E), syntaxin (serotype C) and vesicle-associated membrane protein (VAMP; serotypes $\mathrm{B}, \mathrm{D}$ and $\mathrm{F}$ ), and inhibits the release of acetylcholine to extracellular space $[2,4,5]$. These process cause muscular paralysis in humans and animals.

When pure BoNT is exposed to the digestive conditions with acidic fluid and proteases, the BoNT easily degrades into inactive short peptides [6-15] and thus the pure BoNT exerts the only weak or no oral toxicity. This implies that the pure BoNT seems unlikely to cause the food-borne poisoning, and conflicts with the previous description that the food-borne botulism is the most frequent among three botulism diseases. The answer that resolves this 
discrepancy is the toxin complex (TC). In the culture supernatant and polluted foods, the BoNT is a part of the TC. Serotype A-D strains produce both of hemagglutination-negative and -positive TC, whereas serotype $\mathrm{E}$ and $\mathrm{F}$ strains produce only hemagglutination-negative TC. The hemagglutination-negative TC is $280-\mathrm{kDa}$ M-TC consists of BoNT and nontoxic nonhemagglutinin (NTNHA). On the other hand, hemagglutination-positive TC is $750-\mathrm{kDa}$ L-TC composed of M-TC and three types of hemagglutinins (HAs; HA-70, HA-33 and HA17). Serotype A strain produces additional LL-TC, which might be a dimer of L-TC $[16,17]$.

The oral toxicities of the M-TC, L-TC and LL-TC are much greater than pure BoNT. Therefore it seems that the auxiliary nontoxic proteins play a role for the delivery of the toxin through the animal digestive system so that the botulinum TC exerts the oral toxicity. In our chapter, we describe our findings in the series of studies on the structure and function of botulinum TC, especially focused on the nontoxic proteins NTNHA and HAs.

\section{Assembly pathway of botulinum toxin complex}

On the genome of the $C$. botulinum strains, the gene encoding BoNT forms one or two gene clusters along with other genes (Figure 1) [16,18-22]. In serotypes A-D, cluster 1 contains the genes coding the BoNT and NTNHA, whereas the cluster 2 contains three genes coding HAs. The open reading frames of the genes on the cluster 2 run opposite orientation against those on the cluster 1 . Serotypes E and F lack cluster 2. In the serotypes C and D, the gene designated as $b o t R$ is located on the downstream of the cluster 2 with opposite directions to reading frames of HA genes. Similar genes are also found in the serotypes A and B, and they are located between the cluster 1 and 2. The gene products of bot $R$ do not participate as a constituent of the TC, but control the expression of the genes on the cluster 1 and 2 [23,24].

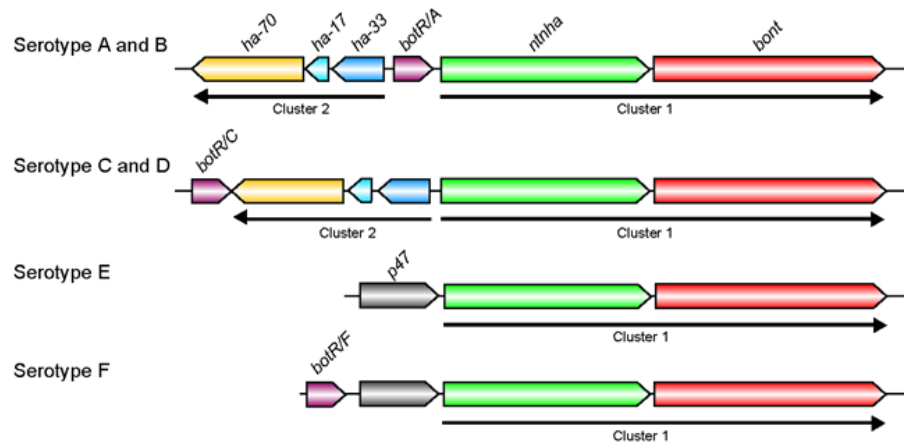

Figure 1. Genetic organization of botulinum neurotoxin related genes of $C$. botulinum. This scheme indicates the BoNT gene clusters in serotype A-F strains. Botulinum TCs are encoded by two gene clusters; cluster 1 encodes BoNT (bont) and NTNHA (ntnha), and cluster 2 encodes three HAs (ha-70, ha17 and $h a-33)$. Each gene cluster is transcribed polycistronically, as indicated by arrows. Genetic organization of serotype A resembles that of serotype B. Similarly, gene organizations of serotype $\mathrm{C}$ and D BoNT clusters are much alike. On the other hand, serotypes E and F do not possess any HA gene. The gene product of botR controls the expression of the genes on the cluster 1 and 2 . In serotypes $\mathrm{E}$ and $\mathrm{F}$, $p 47$ gene is arranged sequentially in the upstream region of the bont gene cluster and its gene product has been uncharacterized. 
Although the C. botulinum serotype $C$ and D strains hold five genes involved in the TC constituents, they produces two types of TCs, M-TC (a complex of BoNT and NTNHA) and L-TC (a complex of M-TC and HAs). Ever since the discovery of TC produced by C. botulinum strains, discrepancy in the coexistence of two types of the TCs in the same culture had been a longstanding mystery. In the culture supernatant, the $150-\mathrm{kDa}$ BoNT of the Land M-TC are split into a 50-kDa light chain (Lc) and a 100-kDa heavy chain (Hc) by the excision of several amino acid residues [25], and the HA-70 in the L-TC is also split into 22$23-\mathrm{kDa}$ and $55-\mathrm{kDa}$ fragments by proteolytic processing after translation [26]. The NTNHA of the M-TC is always found nicked at their N-terminus leading to a $15-\mathrm{kDa} \mathrm{N}$-terminal fragment and a 115-kDa C-terminal fragment, whereas the NTNHA of the L-TC remains intact $[20,27]$. Thus, the components of the TCs are nicked, leading to the appearance of many fragments on the SDS-PAGE. This may complicate the consideration of botulinum TC structure, and hamper the resolution of the discrepancy in coexistence of dual form of TC.

In 2002, we finally found one of the answers to solve this discrepancy [28]. Before that, we needed two breakthroughs to find the answer. First, we serendipitously found a unique serotype D strain 4947 (D-4947), producing the M-TC and L-TC without any nicking in the components of the complex. Second, we have established the method to isolate viable HA components (HA-70, HA-33/HA-17 complex) from the L-TC in the presence of $4 \mathrm{M}$ guanidine hydrochloride [29]. By using the M-TC (BoNT/NTNHA) and isolated HA-70 and HA-33/HA-17 without any nicking obtained from serotype D-4947 strain, we performed reconstitution experiments to construct the L-TC (Figure 2) [28]. Mixture of the M-TC and HA-70 yielded a de novo M-TC/HA-70 complex, whereas a mixture of the M-TC and HA33/HA-17 did not produce any complex. Further the M-TC/HA-70 complex converted to the mature L-TC by mixing with the HA-33/HA-17. On the other hand, M-TC, which contained nicked NTNHA prepared by treatment with exogenous trypsin, could no longer be reconstituted to mature L-TC with HA subcomponents, whereas the L-TC treated with trypsin was not degraded into M-TC and HA subcomponents. Consequently, we concluded that the association of BoNT and NTNHA produces M-TC, and thereafter is converted to the L-TC by assembly with HA-70 and HA-33/HA-17.

\section{Subunit structure of botulinum toxin complex with three extended}

\section{arms}

C. botulinum serotype C and D strains produce 280-kDa M-TC and 750-kDa L-TC. In addition to these TCs, we found three intermediate TC species in the culture supernatants of serotype C and D strain [30,31]. One of them is M-TC/HA-70 complex (490 kDa). Remaining two is $610-$ and $680-\mathrm{kDa} \mathrm{TC}$, corresponding to the intermediate products in the pathway leading from the $490-\mathrm{kDa}$ M-TC/HA-70 to the mature 750-kDa L-TC, which has a smaller number of HA-33/HA-17 complexes than mature L-TC. All of the TC species, except for the 750-kDa L-TC, demonstrated no hemagglutination activity. When the intermediate TC species were mixed with an isolated HA-33/HA-17 complex, all TC species were maturated to $750-\mathrm{kDa}$ L-TC with full hemagglutination activity and had the same molecular composition of native 750-kDa L-TC [30]. Therefore, these findings indicated that the mature L-TC contains multiple HA-33/HA-17 sub-complexes. 


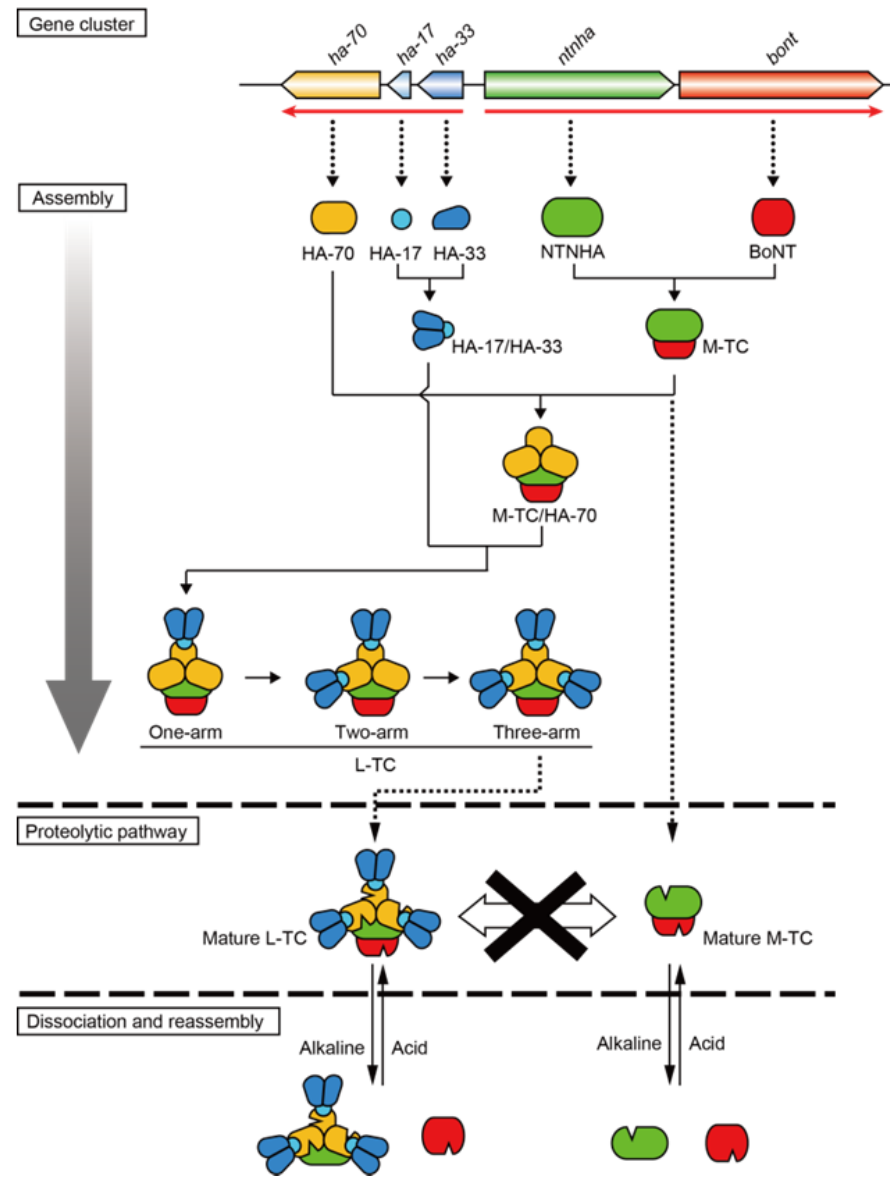

Figure 2. Proposed model for the botulinum toxin complex assembly pathway. Genetic organization of botulinum serotype $\mathrm{C}$ and D TCs and their expressed products are represented based on nucleotide sequences and $\mathrm{N}$-terminal amino acid sequences. The assembly pathway from each gene product and proteolytic pathway is indicated by solid and dotted arrows, respectively. The upper panel represents the assembly pathway of the components to TCs, and the middle panel represents putative proteolytic pathway of the nicked TCs usually observed in serotype $C$ and D strains. The lack of mutual conversion between $\mathrm{L}$ - and $\mathrm{M}-\mathrm{TC}$ is represented by the $\mathrm{X}$ symbol. The lower panel represents dissociation and reassembly of the TCs depending on $\mathrm{pH}$

To characterize the HA-33/HA-17 complex, we determined the X-ray crystal structure of the isolated HA-33/HA-17 complex from D-4947 L-TC at $1.85 \AA$ resolution [17]. As shown in Figure 3, the final model of the complex includes two HA-33 molecules and one HA-17 molecule in the asymmetric unit. This model is consistent with the molecular composition of HA-33/HA-17 complex as determined from molecular mass of the complex by nanoLC/ESITOF-MS yielded a mass of 84,118 Da (calculated molecular mass of two HA-33 plus one HA17 is $84,239 \mathrm{Da})$. 
(a)

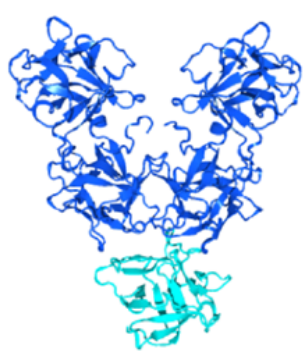

(b)

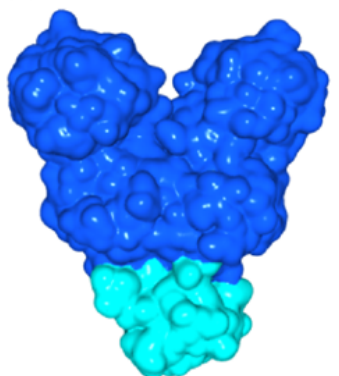

Figure 3. Crystal structure of the HA-33/HA-17 trimer complex. (a) Structure of HA-33/HA-17 trimer complex isolated from serotype D-4947 L-TC as represented by a ribbon diagram. (b) Surface representation of HA-33/HA-17 trimer complex: HA-33 molecules in blue and HA-17 molecule in cyan. Figures were prepared using MolFeat version 3.0 (FiatLux Corp.). The crystal structure of HA-33/HA-17 trimer complex was resolved at $1.85 \AA$.

To clarify the TC structure, the purified TC species, i.e. M-TC, M-TC/HA-70, and 610-, 680- and 750-kDa L-TC, were visualized by negative stained transmission electron microscopy (TEM) [17] (Figure 4). As a result, the M-TC (BoNT/NTNHA) appears as an approximately 13-nm spherical or ellipsoidal particle. The M-TC/HA-70 displayed an acorn-like shape with the HA70 "cone" lying on the M-TC. Interestingly, the 610- and 680-kDa TC and mature 750-kDa LTC revealed unique "arm" attachments that displayed to be rod-like structure. Number of the arm (approximately $10 \mathrm{~nm}$ length) in the TCs was one, two and three for the 610 - and 680-kDa TC and mature 750-kDa L-TC, respectively. We concluded that the arm-like structure is the HA-33/HA-17 that attached to the M-TC/HA-70 complex via HA-70 molecule. At this point,
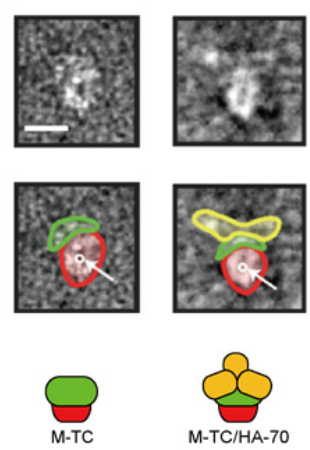
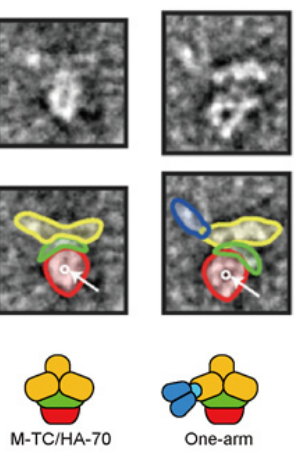
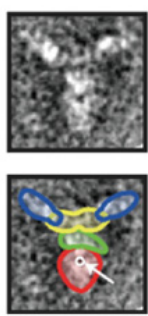
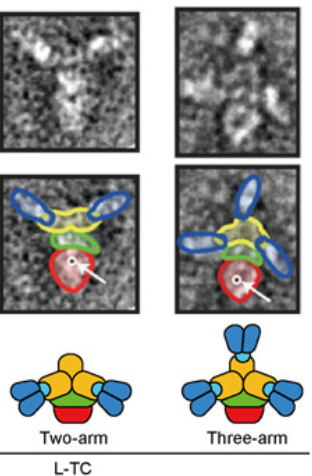

Figure 4. Electron micrographs of the botulinum serotype D-4947 TC species. The upper panel represents the TCs [M-TC, M-TC/HA-70, and one-, two- and three-arm L-TCs, respectively]. Scale bar in the upper left panel indicating $10 \mathrm{~nm}$ applies to all panels. The middle panel represents tracing of images of the subunit components: BoNT in red, NTNHA in green, HA-70 in yellow and HA-33/HA-17 complex in blue. A black spot, which is assumed to be a zinc atom or cavity, is indicated by the circle with an arrow in each BoNT image. The lower panel represents corresponding schematic structure model of each TC. 
the number of the HA-70 molecule in the L-TC is still unclear. Therefore, we examined a densitometric analysis of Coomasie Brilliant blue staining bands on SDS-PAGE gels to understand the number of the HA-70 molecule in the complex. The result indicated that the number of the molecule is three. After our report regarding the number of the HA-70 molecule in the L-TC, the X-ray crystallographic structure of the HA-70 was published [32]. They displayed a three-leaved propeller-like structure, which is consistent with our experiment.

After the TEM observations of the TCs, we named the 610- and 680-kDa TC and mature 750kDa L-TC, as one-, two- and three-arm L-TC [33]. Based on the TEM images and the crystallographic structure of the HA-33/HA-17, we constructed a 14-heteromer model of the mature three-arm L-TC [17]. That is, the three-arm L-TC is composed of a single BoNT molecule, a single NTNHA molecule, and an HA complex. The HA complex is comprised of three HA-70 molecules and three arm structure of HA-33/HA-17 that consists of two HA-33 molecules and a single HA-17 molecule (Figure 5). This model suggests that the six HA-33 molecules exposed to outer of the mature three-arm L-TC, where they play a role in anchoring the complex at the epithelial cell surface.

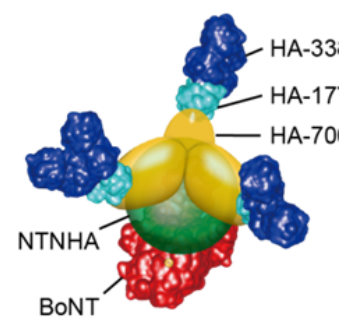

Figure 5. Subunit structure of the botulinum large toxin complex (L-TC). L-TC possesses 14-mer subunit structure and is composed of five types of protein; BoNT, NTNHA and three types of HAs. BoNT molecule is shown in red, NTNHA in green, HA-70 in yellow, HA-33 in blue and HA-17 in cyan, respectively.

\section{Botulinum toxin complex resistant to proteolytic digestion}

BoNT dissociates from the botulinum TC under the alkaline condition, while under the acidic condition the BoNT re-associates with the nontoxic component complex generating the L-TC. Botulinum TC exerts the grater oral toxicity than BoNT detached from the complex. Thus the nontoxic components play a vital role to exert the oral toxicity of the botulinum TC. Of the nontoxic components of the botulinum TC, the NTNHA displays unique property. That is, the NTNHA components in both isolated form and the M-TC (BoNT/NTNHA) complexed form are spontaneously converted to the nicked form leading 15-kDa N-terminal and 115-kDa C-terminal fragments with excision of several amino acid residues at specific sites during long-term incubation [34]. To clarify the role of the NTNHA on oral toxicity of the TC, we constructed the overexpression system that produces the recombinant NTNHA (rNTNHA) in the Escherichia coli strain [15]. The NTNHA gene was amplified by the PCR using the gene specific primer, ligated to pET200-D/TOPO, and then 
transformed into a large-scale E. coli BL21 cell expression system to produce the rNTNHA with His-tagged N-terminus. The rNTNHA was also spontaneously converted to nicked form during long-term incubation generating $\mathrm{N}$-terminal 18-kDa $\mathrm{N}$-terminal (containing His-tag) and 115-kDa C-terminal fragments. Using the rNTNHA, crystallization and preliminary X-ray analysis was successfully performed [35]. Further the rNTNHA was successfully reconstituted with isolated BoNT generating M-TC by mixing these proteins at a molar ratio 1:1 followed by incubation at $\mathrm{pH}$ 6.0. The reconstituted M-TC reversibly dissociated into rNTNHA and BoNT at $\mathrm{pH}$ 8.8. During reconstitution experiment, the intact rNTNHA in the reconstituted M-TC was spontaneously converted into nicked form indicated by the $18-$ and $115-\mathrm{kDa}$ bands on the SDS-PAGE. These features including spontaneous cleavage occurred in the molecule and reconstitution with the BoNT are consistent with those of the native NTNHA.

During the oral delivery of the botulinum TC, the orally ingested TC is exposed to acidity and proteolysis in the gastrointestinal tract. To examine the stability of the rNTNHA, BoNT and reconstituted $\mathrm{M}-\mathrm{TC}$, these proteins were exposed to harsh conditions mimicking environment of the stomach (30 U pepsin in $\mathrm{pH} 2.7)$ and small intestine (1250 U trypsin in $\mathrm{pH}$ 6.0) (Figure 6). The rNTNHA was digested into several fragments after incubation with pepsin for $60 \mathrm{~min}$, whereas trypsin treatment for 360 min yields just nicking in rNTNHA. The BoNT was highly sensitive to both trypsin and pepsin digestion, producing no bands on the SDS-PAGE. In contrast, when the BoNT formed complex with rNTNHA generating the reconstituted M-TC, both proteins exhibited amazing resistance to proteolysis. When the reconstituted M-TC was treated with the pepsin, the BoNT displayed no sign of the fragmentation, while the rNTNHA was converted to nicked form yielding 18- and 115-kDa fragments on the SDS-PAGE. Trypsin treatment of the M-TC produced specific nicked form BoNT and rNTNHA. Because only the reconstituted M-TC displayed high tolerance against both trypsin and pepsin attacks, we presumed that the NTNHA component can alter its conformation to assemble with BoNT, forming an oral toxin that protects BoNT from harsh conditions in the digestive tract. The presumption was certified very recently by the X-ray crystal structure of serotype A M-TC [36].

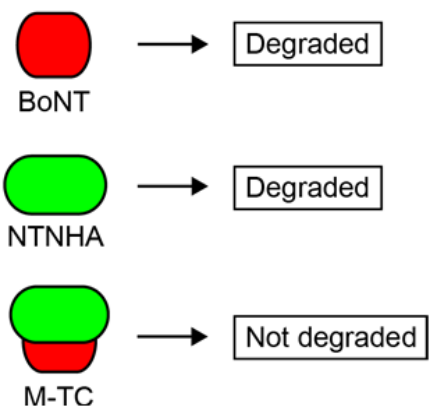

Figure 6. Stability of BoNT, NTNHA and M-TC (BoNT/NTNHA complex) to protease attack. Both BoNT and NTNHA molecules in free form are easily degraded by protease attack, while both proteins in M-TC shows the resistance to pepsin and trypsin. These protease tolerance represents the NTNHA could protect BoNT from protease attack in the digestive tract. 


\section{HA-33 facilitates transport of toxin complex across intestinal epithelial cell monolayer}

For the oral delivery, not only the acidity and proteases in the digestive tract, the physical barrier presented by the intestinal wall is also large obstacle. Of the nontoxic components of the botulinum TC, HA-33 component exposed outermost of the complex, and possesses an ability to recognize sugar chains on intestinal microvilli. Additionally a series of the investigations indicated that the L-TC containing HA-33 components transports across the intestinal epithelial cell monolayer more effectively than pure BoNT $[14,37,38]$. Therefore the HA-33 component appears to play a role in the transport of the TC across the intestinal epithelium. However, some serotype A and serotype E and F strains do not possess the genes that encode the HA components [18,39,40], implying that the absorption of the botulinum TC into intestinal epithelial cells does not depend on the presence of HA components.

In the study of [33], BoNT, M-TC (BoNT/NTNHA), M-TC/HA-70 and three types of L-TC (one-, two- and tree-arm L-TC) with different numbers of the HA-33/HA-17 arm attached were purified from the culture of the D-4947, to examine the cell binding and monolayer transport of serotype D toxins in the rat intestinal epithelial cell line IEC-6. The TCs including pure BoNT were incubated with IEC- 6 cells at $4{ }^{\circ} \mathrm{C}$ for $1 \mathrm{~h}$. Toxins bound to the cells and cytoskeletal actins were visualized by immunohistochemistry using anti-BoNT and phalloidin (Figure 7a). As a result, fluorescence from the BoNT-, M-TC- and M-TC/HA-70-treated cells demonstrated similar intensities, whereas higher intensities were observed after cells were treated with one-, two- or three-arm L-TCs. To quantitatively assess binding of the TCs, toxins bound to the cells were extracted into the sample buffer containing SDS, applied to SDS-PAGE and subjected to Western blot analysis using anti-BoNT. Staining intensities were compared to determine the relative amount of the toxins bound to the cells (Figure 7b). BoNT, M-TC and M-TC/HA-70 displayed similar cell-binding potencies. In contrast, the one-, two- and threearm L-TCs exhibited two, four and five times greater binding than pure BoNT.

Toxin transport through the IEC-6 cell monolayer was examined using the Transwell twochamber system. Toxins suspended in the medium were applied to apical side of the cell monolayer. After 1 to $48 \mathrm{~h}$ incubation at $37^{\circ} \mathrm{C}$, toxins transported through the cell layer were collected from the basal side of the layer, applied to SDS-PAGE and then detected by Western blot analysis using anti-BoNT, anti-NTNHA, anti-HA-70 and anti-HA-33/HA-17 (Figure 8a). The result indicated all TCs including pure BoNT transported through the IEC-6 cell monolayer. L-TC demonstrated greater transport potency than the smaller TCs, whereas pure BoNT, M-TC and M-TC/HA-70 exhibited similar transport efficiencies at all time points (Figure 8b). After a 24-h incubation, the one-, two- and three-arm L-TCs displayed 2, 2.5 and 3 times greater cell monolayer transport, respectively.

To determine the responsible components on the cell binding and cell monolayer transport, pure BoNT, M-TC, M-TC/HA-70 and three-arm L-TC were preincubated with one of the antibodies against their constituents before binding or transport assay (Figure 9). The binding and transport of the pure BoNT were significantly inhibited by preincubation with 
anti-BoNT. Binding and transport of M-TC was significantly inhibited by anti-BoNT, and slightly by anti-NTNHA. As for the M-TC/HA-70, anti-BoNT and anti-HA-70 significantly reduced both the binding and transport of the toxin, while anti-NTNHA had very little effect. Of the antibodies for three-arm L-TC, the HA-33 significantly interfered with both the cell binding and the cell monolayer transport.

(a)

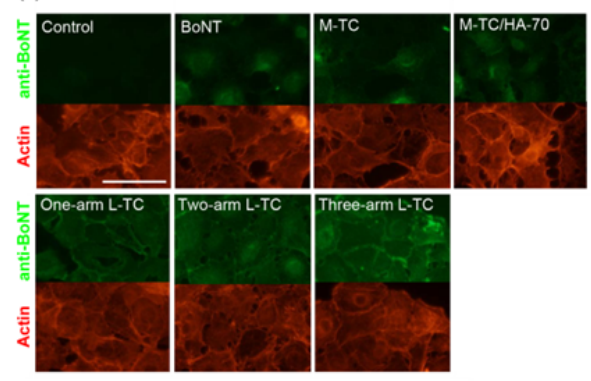

(b)

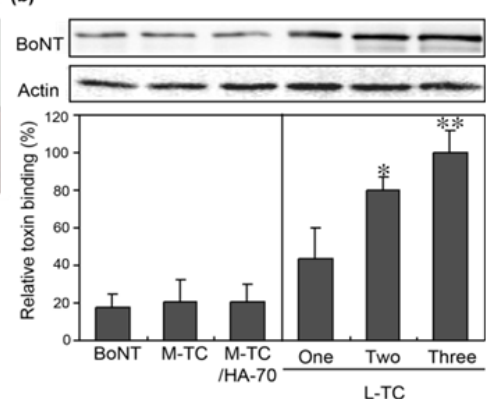

Figure 7. Binding of the BoNT and botulinum TCs to IEC-6 cells. (a) Cells were incubated with BoNT, MTC, M-TC/HA-70, and one-, two- and three-arm L-TCs at $100 \mathrm{nM}$ for $1 \mathrm{~h}$ at $4^{\circ} \mathrm{C}$. Toxins bound to the cells and actins were visualized by immunostaining using BoNT antibody followed by Alexa Fluor 488conjugated secondary antibody (green) and Alexa Fluor 546-conjugated Phalloidin (red). Cells without toxin treatment was employed as a control. Scale bar indicating $50 \mu \mathrm{m}$ at upper left panel applies to all images. (b) Western blot analysis of the binding of the BoNT and TCs to IEC- 6 cells. Cells were incubated with $20 \mathrm{nM}$ TCs. To detect the TCs, BoNT in the cell lysate was detected by using anti-BoNT. Representative data (upper panel) and calculated relative amounts (lower panel) are shown. The relative amounts of the BoNT were calculated based on the intensities of the signals on the Western blot. Double and single asterisk denote a significant increase in binding $(P<0.01$ and $P<0.05)$ compared to the BoNT, respectively.
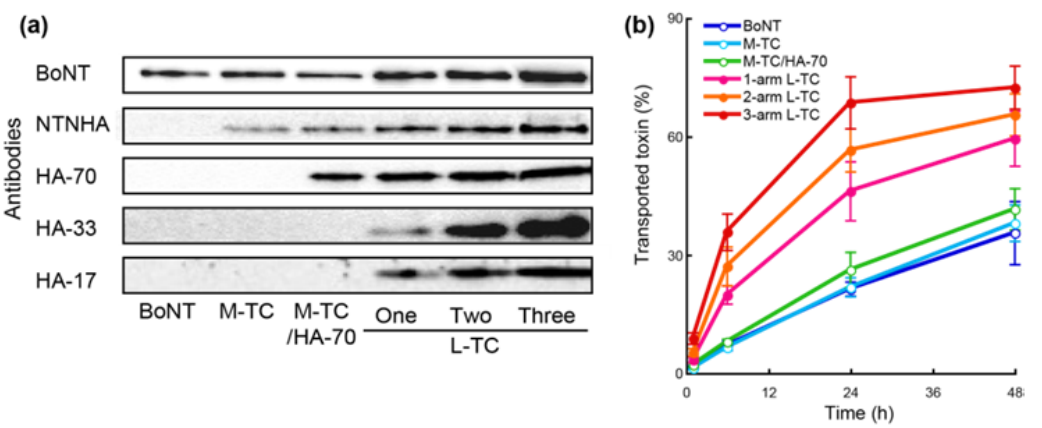

Figure 8. Transport of the BoNT and botulinum TCs through the IEC-6 cell monolayer. (a) Western blot analysis of the transport of TCs through the cell monolayer. Each $20 \mathrm{nM}$ toxin suspension containing $0.2 \mathrm{mg} / \mathrm{ml}$ FITC-dextran (M.W. $500 \mathrm{kDa}$ ) was applied to apical side of the cell monolayer. Transported TCs through the cell monolayer were collected from the medium in the basal side of the layer, and then applied to Western blot analysis. Each component was detected by using corresponding antibody. (b) Time course of toxin transport through the cell layer. Toxin concentration in the basal side medium was estimated from the band intensities of the BoNT on the Western blot. Amounts of the transported toxins are indicated as percentages of the original toxin amounts applied to apical side medium. 


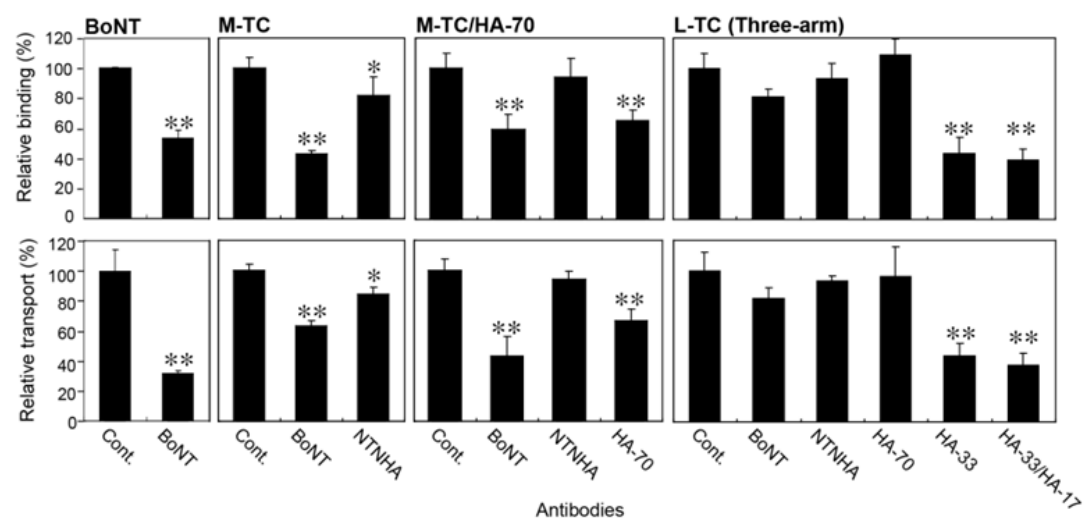

Figure 9. Effect of antibodies against each TC constituents on the cell binding and cell monolayer transport of the botulinum TCs. The toxins $(40 \mathrm{nM})$ were preincubated with antibodies (diluted to 1:10 in incubation buffer) for $1 \mathrm{~h}$ at $37^{\circ} \mathrm{C}$ before cell binding (upper panel) and cell monolayer transport (lower panel) assay. Relative binding and transport were calculated in the same manner for the Figure 8 and compared with the control without antibody preincubation. Double and single asterisk denote a significant increase in binding and transport $(P<0.01$ and $P<0.05)$ compared to the control.

Consequently, all TCs including pure BoNT can transport across the intestinal epithelial cell monolayer via BoNT, NTNHA, HA-70 and HA-33 depending on the TC structure. Nonetheless binding and transport markedly increased with the number of HA-33/HA-17 arms in the TC. We therefore concluded that the HA-33/HA-17 arm is not necessarily required for, but facilitates, transport of the botulinum TC.

\section{Conclusion}

BoNT forms TCs by assembling with nontoxic proteins that include NTNHA and three types of HAs. We provide definitive evidence that NTNHA plays a crucial role in protecting BoNT, which is an oral toxin, from digestion by proteases common in the stomach and intestine. Furthermore, we concluded that the HA-33 is not necessarily required for, but facilitates, transport of botulinum TC. In the food-borne botulism poisoning, the nontoxic components of the TC play a role as a "delivery vehicle" of the unwanted toxic protein. However, we are expecting that the nontoxic proteins would be available for the "delivery vehicle" of the drug and vaccine by substituting the BoNT into functional peptides and proteins.

\section{Author details}

Yoshimasa Sagane, Ken Inui, Shin-Ichiro Miyashita, Keita Miyata,

Koichi Niwa and Toshihiro Watanabe

Department of Food and Cosmetic Science, Faculty of Bioindustry, Tokyo University of Agriculture, Abashiri, Japan 
Tomonori Suzuki

Department of Bacteriology, Okayama University Graduate School of Medicine, Dentistry and Pharmaceutical Sciences, Okayama, Japan

\section{References}

[1] Schiavo G, Rossetto O, Santucci A, DasGupta BR, Montecucco C (1992) Botulinum neurotoxins are zinc proteins. J. biol. chem. 267: 23479-23483.

[2] Montecucco C, Schiavo G (1994) Mechanism of action of tetanus and botulinum neurotoxins. Mol. microbiol. 13: 1-8.

[3] Li L, Singh BR (1999) Structure-function relationship of clostridial neurotoxins. J. toxicol. toxin rev. 18: 95-112.

[4] Schiavo G, Rossetto O, Catsicas S, Polverino de Laureto P, DasGupta BR, Benfenati F, Montecucco C (1993) Identification of the nerve terminal targets of botulinum neurotoxin serotypes A, D, and E. J. biol. chem. 268: 23784-23787.

[5] Dolly JO, Black J, Williams RS, Melling J (1984) Acceptors for botulinum neurotoxin reside on motor nerve terminals and mediate its internalization. Nature 307: 457-460.

[6] Halliwell G (1954) The action of proteolytic enzymes on Clostridium botulinum type A toxin. Biochem. j. 58: 4-8.

[7] Ohishi I, Sugii S, Sakaguchi G (1977) Oral toxicities of Clostridium botulinum toxins in response to molecular size. Infect. immun. 16: 107-109.

[8] Sugii S, Ohishi I, Sakaguchi G (1977) Correlation between oral toxicity and in vitro stability of Clostridium botulinum type A and B toxins of different molecular sizes. Infect. immun. 16: 910-914.

[9] Sugii S, Ohishi I, Sakaguchi G (1977) Intestinal absorption of botulinum toxins of different molecular sizes in rats. Infect. immun. 17: 491-496.

[10] Bonventre PF (1979) Absorption of botulinal toxin from the gastrointestinal tract. Rev. infect. dis. 1: 663-667.

[11] Ohishi I, Sakaguchi G (1980) Oral toxicities of Clostridium botulinum type C and D toxins of different molecular sizes. Infect. immun. 28: 303-309.

[12] Ohishi I (1984) Oral toxicities of Clostridium botulinum type A and B toxins from different strains. Infect. immun. 43: 487-490.

[13] Chen F, Kuziemko GM, Stevens RC (1998) Biophysical characterization of the stability of the 150-kilodalton botulinum toxin, the nontoxic component, and the 900-kilodalton botulinum toxin complex species. Infect. immunol. 66: 2420-2425.

[14] Niwa K, Koyama K, Inoue S-I, Suzuki T, Hasegawa K, Watanabe T, Ikeda T, Ohyama T (2007) Role of nontoxic components of serotype D botulinum toxin complex in permeation through a Caco-2 cell monolayer, a model for intestinal epithelium. FEMS immun. med. microbiol. 49: 346-352.

[15] Miyata K, Yoneyama T, Suzuki T, Kouguchi H, Inui K, Niwa K, Watanabe T, Ohyama T. (2009) Expression and stability of the nontoxic component of the botulinum toxin complex. Biochem. biophys. res. commun. 384: 126-130. 
[16] Inoue K, Fujinaga Y, Watanabe T, Ohyama T, Takeshi K, Moriishi K, Nakajima H, Inoue K, Oguma K (1996) Molecular composition of Clostridium botulinum type A progenitor toxins. Infect. immun. 64: 1589-1594.

[17] Hasegawa K, Watanabe T, Suzuki T, Yamano A, Oikawa T, Sato Y, Kouguchi H, Yoneyama T, Niwa K, Ikeda T, Ohyama T (2007) A novel subunit structure of Clostridium botulinum serotype D toxin complex with three extended arms. J. biol. chem. 282: 24777-24783.

[18] East AK, Bhandari M, Stacey JM, Campbell KD, Collins MD (1996) Organization and phylogenetic interrelationships of genes encoding components of the botulinum toxin complex in proteolytic Clostridium botulinum types A, B, and F: evidence of chimeric sequences in the gene encoding the nontoxic nonhemagglutinin component. Int. j. syst. bacteriol. 46: 1105-1112.

[19] Hauser D, Eklund MW, Boquet P, Popoff MR (1994) Organization of the botulinum neurotoxin $\mathrm{C} 1$ gene and its associated non-toxic protein genes in Clostridium botulinum C 468. Mol. gen. genet. 243: 631-640.

[20] Ohyama T, Watanabe T, Fujinaga Y, Inoue K, Sunagawa H, Fujii N, Inoue K, Oguma K (1995) Characterization of nontoxic-nonhemagglutinin component of the two types of progenitor toxin (M and L) produced by Clostridium botulinum type D CB-16. Microbiol. immun. 39: 457-465.

[21] Fujinaga Y, Inoue K, Shimazaki S, Tomochika K, Tsuzuki K, Fujii N, Watanabe T, Ohyama T, Takeshi K, Inoue K, Oguma K (1994) Molecular construction of Clostridium botulinum type $\mathrm{C}$ progenitor toxin and its gene organization. Biochem. biophys. res. commun. 205: 1291-1298.

[22] Nakajima H, Inoue K, Ikeda T, Fujinaga Y, Sunagawa H, Takeshi K, Ohyama T, Watanabe T, Inoue K, Oguma K (1998) Molecular composition of the 16S toxin produced by a Clostridium botulinum type D strain, 1873. Microbiol. immunol. 42: 599605.

[23] Marvaud JC, Gibert M, Inoue K, Fujinaga Y, Oguma K, Popoff MR (1998) botR/A is a positive regulator of botulinum neurotoxin and associated non-toxin protein genes in Clostridium botulinum A. Mol. microbiol. 29: 1009-1018.

[24] Raffestin S, Dupuy B, Marvaud J, Popoff MR (2005) BotR/A and TetR are alternative RNA polymerase sigma factors controlling the expression of the neurotoxin and associated protein genes in Clostridium botulinum type A and Clostridium tetani. Mol. microbiol. 55: 235-249.

[25] Sagane Y, Watanabe T, Kouguchi H, Sunagawa H, Inoue K, Fujinaga Y, Oguma K, Ohyama T (1999) Dichain structure of botulinum neurotoxin: identification of cleavage sites in types C, D, and F neurotoxin molecules. J. protein chem. 18: 885-892.

[26] Watanabe T, Sagane Y, Kouguchi H, Sunagawa H, Inoue K, Fujinaga Y, Oguma K, Ohyama T (1999) Molecular composition of progenitor toxin produced by Clostridium botulinum type C strain 6813. J. protein chem. 18: 753-760.

[27] Sagane Y, Watanabe T, Kouguchi H, Sunagawa H, Inoue K, Fujinaga Y, Oguma K, Ohyama $\mathrm{T}$ (2000) Characterization of nicking of the nontoxic-nonhemagglutinin 
components of Clostridium botulinum types $\mathrm{C}$ and D progenitor toxin. J. protein chem. 19: $575-581$.

[28] Kouguchi H, Watanabe T, Sagane Y, Sunagawa H, Ohyama T (2002) In vitro reconstitution of the Clostridium botulinum type D progenitor toxin. J. biol. chem. 277: 2650-2656.

[29] Kouguchi H, Watanabe T, Sagane Y, Ohyama T (2001) Characterization and reconstitution of functional hemagglutinin of the Clostridium botulinum type C progenitor toxin. Eur. j. biochem. 268: 4019-4026.

[30] Mutoh S, Kouguchi H, Sagane Y, Suzuki T, Hasegawa K, Watanabe T, Ohyama T (2003) Complete subunit structure of the Clostridium botulinum type D toxin complex via intermediate assembly with nontoxic components. Biochemistry 42: 10991-10997.

[31] Mutoh S, Suzuki T, Hasegawa K, Nakazawa Y, Kouguchi H, Sagane Y, Niwa K, Watanabe T, Ohyama T (2005) Four molecules of the $33 \mathrm{kDa}$ haemagglutinin component of the Clostridium botulinum serotype $\mathrm{C}$ and $\mathrm{D}$ toxin complexes are required to aggregate erythrocytes. Microbiology 151: 3847-3858.

[32] Nakamura T, Kotani M, Tonozuka T, Ide A, Oguma K, Nishikawa A (2009) Crystal structure of the HA3 subcomponent of Clostridium botulinum type C progenitor toxin. J. mol. biol. 385: 1193-1206.

[33] Ito H, Sagane Y, Miyata K, Inui K, Matsuo T, Horiuchi R, Ikeda T, Suzuki T, Hasegawa K, Kouguchi H, Oguma K, Niwa K, Ohyama T, Watanabe T (2011) HA-33 facilitates transport of the serotype $\mathrm{D}$ botulinum toxin across a rat intestinal epithelial cell monolayer. FEMS immunol. med. microbiol. 61: 323-331.

[34] Sagane Y, Watanabe T, Kouguchi H, Sunagawa H, Obata S, Oguma K, Ohyama T. (2002). Spontaneous nicking in the nontoxic-nonhemagglutinin component of the Clostridium botulinum toxin complex. Biochem. biophys. res. commun. 292: 434-440.

[35] Miyata K, Inui K, Miyashita S-I, Sagane Y, Hasegawa K, Matsumoto T, Yamano A, Niwa K, Ohyama T, Watanabe T. (2012) Crystallization and preliminary X-ray analysis of the Clostridium botulinum type D nontoxic nonhaemagglutinin. Acta cryst. F68: 227230.

[36] Gu S, Rumpel S, Zhou J, Strotmeier J, Bigalke H, Perry K, Shoemaker CB, Rummel A, Jin R (2012) Botulinum neurotoxin is shielded by NTNHA in an interlocked complex. Science 335: 977-981.

[37] Fujinaga Y, Inoue K, Watanabe S, Yokota K, Hirai Y, Nagamachi E, Oguma K (1997) The haemagglutinin of Clostridium botulinum type $C$ progenitor toxin plays an essential role in binding of toxin to the epithelial cells of guinea pig small intestine, leading to the efficient absorption of the toxin. Microbiology 143: 3841-3847.

[38] Inui K, Ito H, Miyata K, Matsuo T, Horiuchi R, Ikeda T, Watanabe T, Ohyama T, Niwa $\mathrm{K}$ (2010) Involvement of sialic acid in transport of serotype C1 botulinum toxins through rat intestinal epithelial cells. J. vet. med. sci. 72: 1251-1255.

[39] Sakaguchi G, Sakaguchi S, Kamata Y, Tabita K, Asao T, Kozaki S (1990) Distinct characters of Clostridium botulinum type A strains and their toxin associated with infant botulism in Japan. Int. j. food microbiol. 11: 231-241. 
[40] Johnson EA, Bradshaw M (2001) Clostridium botulinum and its neurotoxins: a metabolic and cellular perspective. Toxicon 39: 1703-1722. 